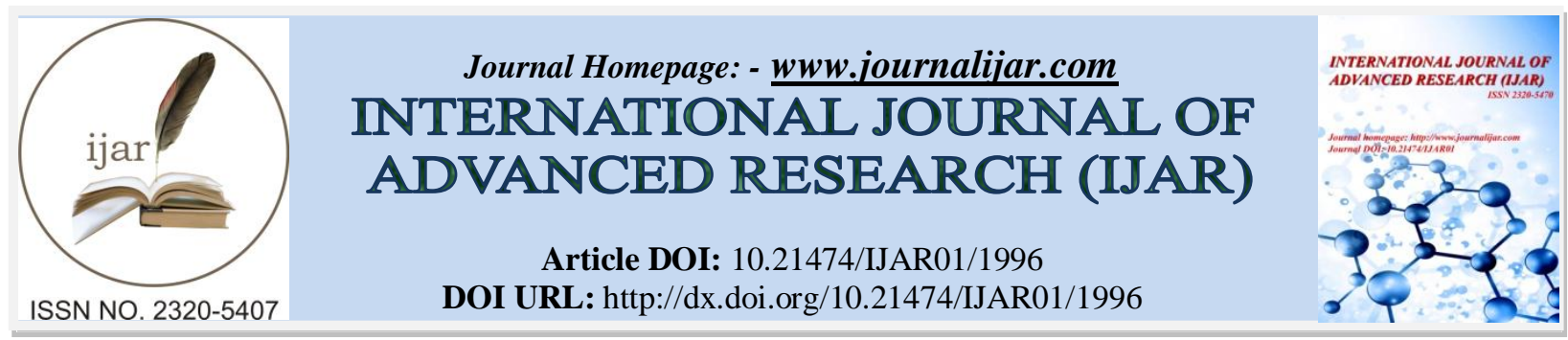

RESEARCH ARTICLE

\title{
WALKING AS AN INCOMPLETE EXERCISE FOR METABOLIC SYNDROME: PHYSIOLOGICAL AND BIOCHEMICAL OVERVIEW
}

\author{
Prasad MV ${ }^{1}$, Mohsina Hyder ${ }^{1}$, Smilty Benny ${ }^{1}$ and Dinesh Roy $D^{2}$. \\ 1. Dr. Prasad's DCRC, Kenichira, Wayanad, Kerala, India. \\ 2. Genetika, Centre for Advanced Genetic Studies, Trivandrum-695024, Kerala, India.
}

\section{Manuscript Info}

Manuscript History

Received: 15 August 2016

Final Accepted: 22 September 2016

Published: October 2016

Key words:-

Lifestyle Disease, Exercise, Diabetes,

Hypertension and Dyslipidemia.

\section{Abstract}

To maintain health and to tackle lifestyle disease, blending of endurance, resistance and aerobic exercise is much better than walking. In walking only a few muscles are working. Because of the absence of glucose-6-phosphatase enzyme in skeletal muscles, glucose stored as glycogen in a muscle cannot be shared by other muscles for energy. So, while walking only a very small quantity of glucose will be burned out as energy. If it burn whole daily dietary intake by exercising whole muscles, maximum glucose can be burned out and for extra energy triglycerides will be used. Reduction in triglycerides will reduce the penetrating power of LDL cholesterol and foam cell formation will be blocked. Increased HDL due to exercise enables plaque stabilization and regression. Increased blood flow achieved will prevent aging, onset and complications of lifestyle diseases and blood pressure. Regular exercise should be a habit (atleast 30 mins), otherwise adherence rate will be less. For lifestyle disease patients regular exercise is mandatory. Exercise will reduce insulin resistance and increase insulin sensitivity. Pre exercise carbohydrate meal will release more insulin through incretin effect. This insulin will refill the energy depleted muscles with glucose, while doing exercise. This will increase the efficiency of exercise. Post exercise carbohydrate + protein meal will enable prompt glycogen synthesis and positive protein balance. Increased blood flow to all parts of the body will reduce hypertension, prevent all the complications of diabetes and will maintain a very good lipid profile.

Copy Right, IJAR, 2016,. All rights reserved.

\section{Introduction:-}

This article is concentrating on tackling lifestyle diseases in a more practical, logical and scientific approach. The main idea behind doing exercise is to burn out the intake calories per day due to the sedentary activities. Human body rapidly maladapts to insufficient physical activity and if continued, results in substantial decreases in both total and quality of life. Lack of physical activity affects almost every cell, organ and system in the body causing sedentary dysfunction and accelerated death. The only valid scientific therapeutic approach to completely counter sedentary dysfunction is primary prevention with physical activity itself. 
There is a belief in the whole society that walking will be enough as a complete exercise to tackle lifestyle diseases like Diabetes, Hypertension and Dyslipidemia. But this review corrects this misbelief. Because of the absence of Glucose-6-phosphatase enzyme in skeletal muscles, glycogen stored in a muscle will not be converted back to glucose, cannot re-enter the blood stream and hence cannot be shared with other muscles. So, out of the 75\% glucose entered the skeletal muscles from daily dietary intake, only very low calories can be burned with walking. Instead of this if do specific exercise for whole skeletal muscles, maximum calories can be utilized. If physical exercise is not enough to burn maximum calories, muscles will be saturated with more glycogen and even if insulin is there in blood it cannot transfer more glucose into the muscles. Glucose and insulin stays in small blood vessels leading to small vessel vacuities and negative feedback towards insulin release due to hyperinsulinemia respectively. Reductions in release lead to reduced production of insulin and leads to prediabetic condition. Only with exercise involving maximum muscles, the condition can be reverted and prevented. By doing even more exercise, triglycerides will be used for energy which reduces its level in the blood. Reduction in triglycerides will reduce the penetrating power of LDL into the plaques and thus more LDL stays in the blood vessels. Increased levels of HDL due to exercise will scavenge more and more LDL from blood vessels and plaques, eventually will lead to good lipid profile and plaque regression. This will bring down aging process as well as will help in the prevention of lifestyle diseases. It also will prevent almost all complications of lifestyle diseases. Exercise will also help the patients to reduce their medications for lifestyle diseases.

\section{Prevalence of Diabetes, Hypertension and Dyslipidemia:-}

According to International Diabetes Federation, most recent estimates indicate that $8.3 \%$ of adults-382 million people have diabetes and the number of people with the disease is set to rise beyond 592 million in less than 25 years. China ranks first in diabetes with 98.4 million among the top ten countries with diabetes. India comes second with 65.1 million and USA next with 24.4 million. The majority of the 382 million people with diabetes are aged between 40 and 59. Every six seconds a person dies from diabetes. Recent reports also indicate that nearly 1 billion adults (more than a quarter of the world's population) had hypertension in 2000 and this is predicted to increase to 1.56 billion by 2025 (Chobanian et al., 2003). The prevalence of hypertension in the late nineties and early twentieth century varied among different studies in India, ranging from 2-15\% in urban India and 2-8\% in rural India. In India, there has been an alarming increase in the prevalence of cardio vascular disease over the past two decades so much so that accounts for 24\% of all deaths among adults aged 25-69 years. Asian Indians have been found to develop CVD at a younger age than other population. In 2005, the World Health Organization (WHO) has estimated that 49 per cent of all deaths have been attributed by Lifestyle diseases.

\section{Etiology of Lifestyle Disease:-}

Type 2 diabetes results from a combination of the inability of muscle cells to respond to insulin properly (Insulin resistance) and inadequate compensatory insulin secretion. The decreased insulin stimulated glucose uptake is due to impaired insulin signaling and multiple post receptor intra cellular defects including impaired glucose transport, glucose phosphorylation, reduced glucose oxidation and glycogen synthesis (Bajaj et al., 2003). Type 2 diabetes occurs when a diabetogenic lifestyle (Excessive calories, inadequate exercise and obesity) is superimposed upon a susceptible genotype. Though the exact causes of hypertension are usually unknown, there are several factors that have been highly associated with the condition. These include Obesity, Diabetes, Sedentary lifestyle, Lack of physical activity, Smoking, Stress, Aging, High salt intake etc. More than this if exercise is lesser than the intake calories, LDL gets accumulated in the blood vessel walls throughout the body and throughout life. This increases the peripheral resistance and only if heart pumps much more forcefully oxygen, water and nutrition will reach all peripheral cells. That is why with aging BP goes high and is normal physiology. If this rise in BP is suppressed unnecessarily with medicines, the person may go for early aging and accelerated all death. But this can be tackled easily with exercise involving maximum muscle groups and reduced calorie intake. The Framingham heart study showed that a weight gain of 5\% increases the risk of hypertension by $30 \%$ in a 4 year period. Patients with diabetes and obesity typically have an artherogenic dyslipidemia characterized by elevated triglycerides and low HDL levels as well as an increased proportion of small dense LDL particles. Abnormalities of lipoprotein lipase activity, hormone sensitive lipase activity and fatty acid metabolism all contribute to baseline and often extreme post prandial hypertriglyceridemia associated with type 2 diabetes and insulin resistance. Although fasting Lipoprotein lipase (LPL) levels are typically increased in obese subjects because of the large number of adipocytes, insulin resistance at the level of the fat cell causes decreased LPL activity and therefore an abnormal response of LPL to glucose load (Krentz, 2003). Diminished LPL activity leads to an accumulation of artherogenic LDL precursors such as VLDL in the circulation. The likely causes for the increase in the cardio vascular disease rates include lifestyle changes 
associated with urbanization and the epidemiologic and nutritional transitions that accompany economic development.

\section{Why walking is not enough?}

Walking, as a typical low impact exercise is the most popular and most preferred exercise among patients with type 2 diabetes (Thomas et al., 2004). In walking, only few muscles are working where glucose utilization and glycogen depletion will be minimum. Because of the absence of glucose-6-phosphatase enzyme in muscles, glycogen depleted in the muscles will be refilled only from the blood glucose. Glycogen store from other muscles will not be shared by calf muscles, the main muscles used while walking. In walking, only few muscle groups like calf muscles (Gastrocnemius and soleus), thigh muscles and shoulder girdle muscles are working. Energy expended by running in the runners was more than twice that reported for walking by walkers. The runners had $38 \%$ lower risk for incident hypertension, 36\% lower risk for hypercholesterolemia and 71\% lower risk for diabetes mellitus than walkers (Paul et al., 2013). The benefits of physical activity depend on three elements: the intensity, duration, and frequency of exercise. Because walking is less intensive than running, patients have to walk for longer periods, get out more often, or both to match the benefits of running. In areas with prolonged monsoon both walking and running will be less by $90 \%$ or so. Hence exercise involving move muscle groups inside a room will be much more preferable.

\section{Lifestyle Disease Management:- Planning the Diet:-}

To increase the efficacy of exercise session, pre exercise carbohydrate intake should be planned. If it is a direct glucose, this will stimulate Incretins of the gut and will release insulin from the beta cells of pancreas. This insulin will transfer more and more glucose from the blood stream into the muscles as it is utilized during exercise. Similarly post exercise carbohydrate and protein intake will replenish glycogen stores and net positive protein balance. In diabetics, exercise will reduce insulin resistance, increase insulin sensitivity; muscle membrane glucose transport capacity and health related quality of life. According to National Heart Foundation of Australia's national blood pressure and vascular disease advisory committee- "Regular exercise is the first treatment recommended to lower blood pressure and improve cardio vascular health both in general population and in those people with hypertension. Regular physical activity makes the heart stronger. A stronger heart can pump more blood with less effort. If we can perform more exercise than which is required to burn daily dietary calories from carbohydrate and fat, the triglycerides (TGL) level can be kept low which reduces the penetrating power of LDL in to the plaques. As more LDL stays in blood stream, liver produce more nascent HDL to clean this LDL and reverse cholesterol transport will be enhanced. In the long run this will reduce the plaque size because of the increased HDL activity. This will reduce peripheral resistance and BP will be lowered, Lipid profile will be shifted more towards HDL than TGL and LDL. If the heart has to work less, the force on the arteries decreases, lowering the blood pressure. Judicious planning of diet and exercise thus will be the answer for almost all the health problems of mankind.

\section{Nutrition therapy:-}

A healthy eating pattern, regular physical activity and often pharmacotherapy are key components of lifestyle disease management. Patients with diabetes hypertension and dyslipidemia often require caloric restriction to promote weight loss. According to American Diabetes Association (ADA) there is not a "one size fits all" eating pattern for individuals with diabetes. The ADA also recommends that people at high risk for type 2 diabetes eat high-fibre (14g fibre for every 1000 calories) and whole grain foods. Urinary glucose excretion was also lowered by high-fibre diet because of slow and sustained absorption from intestine which prevents the sudden hike in blood sugar which in turn reduces the urinary excretion of sugar. This reduces the renal complications to a certain extent. A meal plan that is moderate in carbohydrates, low in saturated fat and rich in proteins is always recommended. Bed time and between meal snacks are not usually recommended for lifestyle disease patients. The Dietary Approaches to Stop Hypertension (DASH), a diet regimen rich in fruits and vegetables and low fat dairy products has been proven to significantly reduce BP (Sacks et al., 2001). Increased intake of soluble fiber in the form of Oat bran, Pectins, certain gums and psyllium products can reduce total and LDL cholesterol by $5 \%$ to $20 \%$. Fish oil supplementation has a fairly large effect in reducing triglycerides and LDL cholesterol. People typically reduce fat intake in an effort to lower cholesterol levels, but without appropriate nutritional counseling, individuals will often increase carbohydrate intake as they reduce dietary fat. Carbohydrates raise triglyceride levels and lower HDL levels, usually without an added effect on LDL. After using carbohydrate for energy, body uses TGL for energy through glucogon mediated hormone-sensitive lipase (HSL) activity. 


\section{Exercise and Glycogen Stores:-}

Glycogen is the storage form of carbohydrates in humans and other mammals. The use of electron microscopy has revealed that glycogen is found as discrete glycogen particles located in distinct pools within the fibres. Each glycogen granule has its own metabolic machinery with glycolytic enzymes and regulating proteins (Wanson and Drochman, 1968). The main function of glycogen is to maintain a physiological blood glucose concentration. In humans, approximately $75 \%$ of the glycogen is stored in skeletal muscles, as skeletal muscles account for approximately $40-50 \%$ of body weight in healthy population and the glycogen concentration is $80-150 \mathrm{mmol} \mathrm{kg}$ $\mathrm{ww}^{-}{ }^{-}$. While liver has a higher glycogen concentration, but as the liver is much smaller approximately $1.5 \mathrm{~kg}$ and the total amount of liver glycogen is approximately 100 grams. Other tissues like the heart and brain contains minor glycogen stores with important physiological function (Taylor et al., 1996). As need arises, liver glycogen directly contributes to blood and maintains its normal glycemic levels. But skeletal muscles are unable to release glucose to blood due to lack of glucose 6 phosphatase enzyme. Instead, Glucose-6-phosphate will be converted to Fructose-6 phosphate by Phospho glucose isomerase and enter glycolytic pathway and used for energy in aerobic condition. Indeed, muscle glycogen can be broken down to lactate, which can be transported to the liver and via Gluconeogenesis in the liver contribute to maintaining euglycemia through Cori's cycle. The glycogen content in skeletal muscles from obese and type 2 diabetes subjects is comparable to lean subjects or may even be reduced (Shulman et al., 1990).

During exercise, glycogen in the muscles will be used for energy and when more and more glucose moieties gets depleted from glycogen, more GLUT4 containing vesicles will be free in the sarcoplasm as these vesicles are structurally attached to the glycogen molecules thus more glucose will be translocated. The glycogen synthesis will also be enhanced. Usually insulin is the hormone which facilitates this action. Even in the absence of insulin, MAPK pathway will be activated with exercise and more glucose enters the muscle for glycogen synthesis. Exercise induced, contraction-mediated GLUT4 translocation to the muscle membrane is independent of insulin and occurs through calcium dependent protein kinase IV and secondarily through AMPK, which induces expression of PGC-1 alpha, a transcriptional co activator that is essential for mitochondrial biogenesis. Glycogen synthesis following exercise occurs in two distinct phases-rapid phase and slow phase. The rapid phase lasts approximately 30-60 minutes (Maehlum et al., 1977) and slow phase for several hours if carbohydrate availability is high and insulin levels remain elevated (Ivy et al., 1991). Decreasing glycogen content by exercise or fasting stimulates glycogen accumulation to levels above the glycogen content in well fed conditions (Hespel and Richter, 1990). Glycogen is a vital fuel source for high intensity and prolonged exercise and the dependence of this energy substrate increases as exercise intensity rises (Bergstrom and Hultman, 1966).

\section{Benefits of exercise:- \\ Regular exercise:-}

Regular exercise is a key element in the management of type 2 diabetes (Schellenburg et al., 2013) has also been reported to be effective in the prevention and delay of onset of type 2 diabetes. Regular exercise increases insulin sensitivity and ameliorates glucose metabolism (Derouich et al., 2002). Regular physical activity has been shown to improve psychological well being and self esteem (Kenneth, 1999) and also improves perceived health related quality of life and mood status of type 2 diabetic subjects (Nicolucci et al., 2012). Regular exercise can strengthen antioxidant defences and may reduce oxidative stress (Kim et al., 1996). Physical activity guidelines recommend that adult should accumulate 30 mins or more of moderate intensity physical activity on most, preferably all, days of the week (Pate et al., 1995).

When regular exercise exceeds the daily calorie intake blood sugar comes down gradually. When it comes below 90 $\mathrm{mg} / \mathrm{dl}$, glucagon will be released from the alpha cells of pancreas. This will stimulate hormone sensitive lipase and activate lipolysis, releasing more free fatty acids which will be used for energy. More and more free fatty acids will be released due to lipoprotein lipase activity and triglyceride gradually comes down. When triglycerides are reduced, the LDL molecules enlarge in size and density will be reduced. Penetrating power through endothelial walls will be decreased. Now more LDL stays in the smaller blood vessels. Increase in exercise count gradually raises the HDL level which in turn scavenges more and more LDL from the small vessels through reverse cholesterol transport. After few months triglycerides and LDL will be reduced and the HDL levels will be raised. Reverse cholesterol transport will clear the blood vessels from bad cholesterol, which in turn reduces the chance of arthrosclerosis and thereby cardiac accidents.

Similarly, a fall in blood pressure may be related to weight loss and relaxation produced by exercise. Postulated mechanisms for the reduction of both systolic and diastolic blood pressure that occurs with regular exercise training 
include the following- Reduced visceral fat, Improved sodium elimination due to altered renal function, Reduced plasma renin and catecholamine activity and reduced sympathetic and increased para sympathetic tone. Increased HDL activity due to exercise lead to (plaque regression) reduction in size of multiple blocks in the blood vessels, $(\mathrm{PR})$, reduces peripheral resistance in blood vessels, increased perfusion to cells lead to reduction in BP.

\section{Planned exercise:- \\ Benefits of pre-exercise:- \\ Carbohydrate intake - (include protein intake):-}

During an overnight fast, more glycogen will be used in the heart, lungs and brain while sleeping, for its activities. Similarly liver glycogen stores are reduced substantially, with studies reporting glycogenolysis rates of approximately $0.2-0.3 \mathrm{mmol}$ glucosyl units per min during the fast which equates to an approximate $80 \%$ reduction in liver glycogen stores overnight (Jeukendrup et al., 2010). Blood glucose and up to even $80 \%$ of liver glucose will be used for energy while sleeping overnight. So while doing exercise in the morning, only less glucose will be available in the blood stream for glycogen synthesis. If we take a pre exercise carbohydrate meal, it will transfer more glucose into the blood stream. Thus blood glucose and liver glycogen will be replenished. Recent research indicates that the type and composition of carbohydrate can influence gastric emptying, fluid delivery, absorption and gastro intestinal distress. Studies examining the effects of consuming glucose/ maltodextrin + fructose (GF) during exercise have reported enhanced gastric emptying, fluid delivery and absorption rates versus isocaloric amounts of glucose alone (Jeukendrup et al., 2008). These effects may be due to non-competitive carbohydrate intestinal transport, as glucose and maltodextrin are transported into the blood stream via the sodium dependent SGLT1 transporter and fructose via GLUT 5. This multiple transport of carbohydrate seems to enhance the delivery of more carbohydrate into the muscle (Shi et al., 1995). Consumption of carbohydrate prior to exercise can maximize glycogen storage. With the use of NMR spectroscopy Taylor et al 1996 reported that following the consumption of a mixed meal, approximately $20 \%$ of ingested carbohydrate is directly stored as liver glycogen (Taylor et al., 1996). Moreover Coyle and colleagues reported a $42 \%$ increase in muscle glycogen storage following pre-exercise ingestion of carbohydrate. Therefore consuming carbohydrate may help to increase carbohydrate availability by maximizing carbohydrate fuel stores prior to exercise (Coyle et al., 1985). This pre exercise carbohydrate meal will stimulate beta cells of the pancreas through incretin effect and more insulin will be released into the blood stream. While doing exercise in the morning, when more and more glucose is used for energy from the glycogen pool of the muscles more and more glucose will be translocated into the sarcoplasm with the help of released insulin. In case of insulin resistance also, exercise will enhance MAPK pathway which even in the absence of insulin also, facilitate glycogen synthesis. Gradually insulin resistance also reduced with exercise as more and more GLUT 4 transporters are set free in the sarcoplasm with depletion of glycogen for more energy.

\section{Benefits of post exercise:-}

Carbohydrate and protein intake:-

A supplementation of protein and /amino acid following exercise is the best for net positive protein balance that enables skeletal muscle hypertrophy. Carbohydrates are essential to replenish glycogen stores soon after prolonged or high intensity exercise. Koopman and colleagues, (2007) found supporting evidence, as they examined the effects of ingesting different amount of carbohydrates with adequate protein intake on post exercise protein synthesis. The addition of protein and amino acids to a carbohydrate solution increases net muscle protein synthesis to a higher degree than carbohydrates alone (Koopman et al., 2007). If a carbohydrate rich food is given soon after a heavy exercise session, Incretin effect will release more insulin into the blood stream which transfers more glucose into the glycogen depleted muscles through phosphatidylinositol signalling pathway. Thus incretin effect will also be responsible for rapid phase glycogen synthesis in the muscles.

\section{Cardio vascular benefits:-}

With regular exercise the cardiac muscle in the heart hypertrophies resulting in thicker stronger walls and therefore increases in heart volumes. The more blood pumped around the body/min, the faster oxygen is delivered to the working muscles. Arterial walls become more elastic which allows greater tolerance of changes in blood pressure. The density of the capillary beds in the muscles and surrounding the heart and lungs increases as more branches develops. This allows more efficient gaseous exchange of oxygen and carbon dioxide. The number of RBCS increases, improving the body's ability to transport oxygen to the muscles for aerobic energy production. The accumulation of lactic acid is much lower during high levels activity, due to the circulatory system providing more oxygen and removing waste products faster. Regular moderate intensity exercise prevents blood clotting (anti 
thrombotic) and helps to maintain a normal heart rhythm (anti arrhythmic) in people with Coronary heart disease (CHD). The anti thrombotic effect of chronic exercise is also reversible and will return to previous values within 4 weeks of exercise cessation (Saeid et al., 2012).

\section{Biochemical benefits:- \\ Relation with Insulin resistance and sensitivity:-}

In obesity and type 2 diabetes, fatty acid metabolism in the skeletal muscle is dysregulated, resulting in the accumulation of lipids within the muscle cell. These intra muscular lipid products interfere with insulin signalling within the muscle cell there by contributing to insulin resistance (Shulman, 2000). In insulin resistant states, insulin stimulated glucose uptake is markedly reduced in skeletal muscle. In aging and insulin resistant conditions the ability of insulin to stimulate GLUT 4 translocation decreases resulting in a reduced GLUT4 content at the plasma membrane (Zierath et al., 2002). As glycogen storage capacity is reduced in the muscles of insulin resistant subjects will cause a stronger feedback inhibition of glycogen synthase and thus deteriorate glucose regulation.

Exercise improves insulin sensitivity in both healthy subjects and insulin resistant people (Heath et al., 1983). The flux by which glucose is removed from the blood into the skeletal muscle glycogen is the major determinant of insulin sensitivity (Hojlund and Becknielsen, 2006). Muscle glycogen synthase activity and glucose transport as increased after exercise, an enhanced metabolic action of insulin in skeletal muscle including glycogen synthesis is also observed. The duration of this period with enhanced insulin sensitivity may last up to 48 hours depending upon the rate of muscle glycogen repletion. Moderate intensity exercise can be sustained for a prolonged time and training planned on moderate intensity/long distances increases insulin sensitivity up to 14 days after the end of the last exercise session and favours lipid consumption (Bajpeyi et al., 2009). Regular exercise improves perceived health related quality of life and mood status of type 2 diabetic subjects (Pedersen et al., 2006). Physical activity can help people with diabetes to achieve increased cardio respiratory fitness, increased vigour and maintenance of weight loss (Chudyk et al., 2011).

\section{Relation with Hypertension:-}

Besides improving glycemic control, a recent meta-analysis showed that structured exercise intervention studies in non insulin dependent type 2 diabetes patients reduce systolic blood pressure with $-4.16 \mathrm{~mm} \mathrm{Hg}$ (Thomas et al., 2006). Such reductions in mean arterial blood pressure are clinically relevant and are similar to the effects of add-on blood pressure lowering therapy using a combination of an ACE inhibitor and Thiazide diuretic (Patel et al., 2007). Prospective studies have demonstrated that regular physical activity is associated with a significantly reduced risk of hypertension in men and women independent of age, education, smoking habits, alcohol intake, history of diabetes, BMI and SBP at baseline (Hu et al., 2004). It has been hypothesized that increasing physical activity might reduce BP through decreased body weight or favourable changes in body fat distribution. The Finnish Diabetes Prevention Study showed that over weight subjects with glucose intolerance who received intensified lifestyle intervention, which consisted of diet and moderate exercise for at least $30 \mathrm{~min} /$ day resulted not only in a marked reduction in the risk of developing type 2 diabetes, but also in a significant drop in BP (4mm $\mathrm{Hg}$ for SBP and $2 \mathrm{~mm} \mathrm{Hg}$ for DBP compared with control subjects) (Tuomilehto et al., 2001).

\section{Relation with Dyslipidemia:-}

Daily aerobic exercise is an important lifestyle modification that can improve the lipid profile in patients with type 2 diabetes or insulin resistance, especially if exercise is combined with appropriate dietary recommendations to achieve weight loss. Exercise increases skeletal muscle Lipoprotein lipase (LPL) activity which lowers triglycerides levels (Gill et al., 2003). When circulating triglycerides are reduced, there is reduced CETP (Cholesterol ester transfer protein)- mediated exchange of lipid from triglyceride rich particles to HDL particles. HDL particles with lower triglyceride content are less vulnerable to clearance and therefore the HDL level increases. Exercise also causes increased formation of HDL Cholesteryl esters and decreased hepatic lipase activity, both of which contribute to increased HDL levels.

\section{Physiological benefits:-}

Advantages of muscle specific exercises:-

In lifestyle diseases, instead of walking, muscle specific exercises should be planned to burn out maximum glycogen stores. When exercise exceeds the calorie intake, along with blood sugar lipid profile also comes to normal. Instead of utilizing the glycogen stored in few muscles like calf muscles and thigh muscles continuously. Glycogen stored in whole muscle groups in body should be worked out moderately through a planned and structured exercise pattern 
including combination of aerobic, resistance and endurance type exercises. Since aerobic exercise improves oxygen consumption and increases the functioning of the cardio vascular and respiratory systems. Aerobic exercise also improves the physiological parameters including glycemic control, fasting blood glucose level and lipid profile (these are biochemical parameters). Moreover it can restore the endothelial function and reduces the arterial stiffness which is the positive denominator for developing cardio vascular complications in type 2 diabetes mellitus (Stewart et al., 2002). High volume aerobic exercise produced weight loss also improves insulin sensitivity (Ross et al., 2000). Resistance exercise has been reported to enhance insulin sensitivity, daily energy expenditure and quality of life (Poehlman et al., 2000). Resistance exercise includes increases in glycolytic capacity and up regulation of proteins in the insulin signalling cascade (Holten et al., 2004). Resistance exercise alone can improve blood pressure, lipid levels and reduce adiposity (Cornelissen et al., 2011). Two large randomized trials among individuals with type 2 diabetes also suggest that the combination of aerobic exercise and resistance exercise results in greatest improvement in glycemic control compared with either type of activity alone (Sigal et al., 2007).

According to hippocrates: "All parts of the body, if used in moderation and exercised in labours to which each is accustomed, become there by healthy and well developed and age slowly; but if they are unused and left idle, they become liable to disease, defective in growth and age quickly".

\section{Conclusion:-}

The popular belief in the society is that walking is a very good exercise. But a medical person is often advising walking as an exercise, as the new generation is not doing even that. Main intention of exercise is to burn out the daily calorie intake. Energy expenditure while jogging is more than double that of walking.

After an overnight fast, even up to $80 \%$ of the liver storage of glycogen is utilized for basal metabolism and for all basal activities during sleep. Out of the $75 \%$ glucose entering the muscle to store as glycogen, evenly distributed to all skeletal muscles, only very small quantity will be utilized for energy while walking. As glycogen store in a muscle will not be shared by other muscles because of the absence of glucose 6 phosphatase enzyme in skeletal muscles. In the case of diabetics, because of the reduced insulin activity, even the entry of blood sugar to the calf muscles will not be prompt as well as the utilization of liver glycogen storage. So a diabetic patient will be tired fast if he use small group of muscles for energy burning, like walking.

Exercise should be planned in such a manner to work out maximum skeletal muscles as in endurance type of exercise. Resistance exercise will burn out maximum calories and aerobic exercises will be the best for cardiac and respiratory systems. So mixing endurance, resistance and aerobic exercise judiciously will be the best. Regular exercise daily for atleast 30 mins will be the best for maximum calorie utilization and for maintaining health. Extra exercises than the daily dietary intake will utilize fatty acids from triglycerides for extra energy. This reduction in triglyceride level eventually will reduce the penetrating power of LDL cholesterol, leading to reduced entry of LDL cholesterol into the plaques. LDL will be increased in the blood vessels and to scavenge all this LDL, more HDL will be produced in the liver. This HDL cholesterol will scavenge the LDL cholesterol even from the foam cells of the plaque causing plaque regression. This will increase the blood flow to all organs and will help to prevent all the complications of diabetes, hypertension and hypercholesterolemia as well as the aging process. Continuous walking in obese people will damage the knees leading to osteoarthritis especially in the aged group. So, diet control (not restriction) and planned exercise is the best to maintain health, to (reduce) prevent lifestyle diseases, to prevent all the complications of lifestyle diseases and aging process.

\section{References:-}

1. Akiko S Hosler., Mary P Gallant., Mary Riley-Jacome., Deepa T Rajulu. (2014). Relationship between objectively measured walk ability and exercise walking among adults with Diabetes. Journal of Environmental and Public Health; Volume 2014; Article ID 542123.

2. Alison B Evert., Jackie L Boucher., Marjorie Cypress. (2013). Nutrition therapy recommendations for the management of adults with Diabetes. Diabetes care; Volume 36.

3. Anders Grontved., An Pan., Rania A Mekary., Meir Stampfer., Walter C Willet., Joann E Manson., Frank B Hu. (2014). Muscle strengthening and conditioning activities and Risk of type 2 diabetes: A Prospective study in two cohorts of US Women; Plos medicine; Volume 11; Issue 1; e1001587.

4. Bajpeyi, S., Tanner, C.J., Slentz, C.A., et al. (2009). Effect of exercise intensity and volume on the persistance of insulin sensitivity during training cessation; J Appl Physiol; 106; 1079-1085.

5. Barbara Strasser, and Dominik Pesta. (2013). Resistance training for Diabetes Prevention and therapy: Experimental findings and molecular mechanisms; Biomed Research International; Volume 2013; Article ID 805217. 
6. Bergstorm, J., and Hultman, E. (1966). Muscle glycogen synthesis after exercise, an enhancing factor localized to the muscle cells in Man; Nature 210; 309-310.

7. Canadian Diabetes Association Clinical Practice Guidelines. (2013). Physical activity and Diabetes; Canadian Journal of Diabetes; 37; S40-S44.

8. Chobanian, A.V., Bakris, G.L., Black, H.R., et al. (2003). The Seventh Report of the Joint National Committee on Prevention, Detection, Evaluation and Treatment of high blood pressure: the JNC 7 Report; JAMA 2003 ; 289 (19): 2560-2572.

9. Chris poole, Colin Wilborn, Lem Taylor and Chad Kerksick. (2010). The role of post exercise nutrient administration on muscle protein synthesis and glycogen synthesis; Journal of Sports Science and Medicine 9; 354-363.

10. Chudyk, A. and Petrella, R.J. (2011). Effects of exercise on cardio vascular risk factors in Type 2 Diabetes- a meta analysis; Diabetes care 2011; 34; 1228-37.

11. Cornelissen, V.A., Fagard, R.H., Coeckel berghs, E., and Vanhees, I. (2011). Impact of resistance training on blood pressure and other cardio vascular risk factors; Hypertension 58; 950-958.

12. Coyle, E.F., Coggan, A.R., Hemmert, M.K., Lowe, R.C., Walters, T.J., (1985). Substrate usage during prolonged exercise following a pre exercise meal; J Appl Physiol 1985; 59; 429-433.

13. Derouich, M., and Boutayeb, A. (2002). The effect of physical exercise on the dynamics of glucose and insulin; J Biochem 2002; 35 (7); 911-917.

14. Fox, K.R. (1999). The influence of physical activity on mental well-being; Public health nutrition; 2 (3a); $411-418$.

15. Murtagh, E.M., Boreham, C.A., and Murphy, M.H. (2002). Speed and exercise intensity of recreational walkers. Preventive medicine. 31;35(4):397-400.

16. Elaine M Murtagh, Colin AG, Boreham and Marie H Murphy. (2002). Speed and Exercise intensity of Recreational walkers; School of Applied Medical Sciences and Sports Studies; Ireland 35(4):397-400.

17. Erick A Ritcher., Wim Derave., and Jorgan FP Wojtaszewski. (2001). Glucose Exercise and Insulin; Emerging Concepts; Journal of Physiology. 535.2; PP 313-322.

18. Frank W Booth., Christian K Roberts., and Mathew J Laye. (2012). Lack of exercise is a major cause of chronic diseases; Compr Physiol; April 2(2); 1143-1211.

19. Gill, H.M., and Hard man, A.E. (2003). Exercise and post prandial lipid metabolism; an update on potential mechanisms and interactions with high carbohydrate diets; J Nutr Biochem 2003; 14; 122-132.

20. Shulman, G.I. (2000). Cellular mechanisms of insulin resistance; Journal of clinical investigation; Vol 106; No:2; PP 171-176.

21. Hayley Neill, M.O. (2013). AMPK and Exercise: Glucose uptake and Insulin Sensitivity; Diabetes Metab J 37; 1-21.

22. Heath, G.W., Gavin. J.R., Hinder-Liter, J.M., Hagberg, J.M., Bloom field, S.A., and Holloszy, J.O. (1983). Effects of exercise and lack of exercise on glucose tolerance and insulin sensitivity; J Appl Physiol 55; 512-517.

23. Hespel, P., and Richter, E.A. (1990). Glucose uptake and transport in contracting perfused rat muscle with different pre contraction glycogen concentrations; J Physiol (Lond) 427, 347-359.

24. Holten, M.K., Zacho, M., Gaster, M., Juel, C., Wojtaszewski, J.F.P., et al. (2004). Strength training increases insulin mediated glucose uptake, GLUT 4 content and insulin signalling in skeletal muscle in patients with type 2 diabetes; Diabetes 53; 294-305.

25. Hojlund, K., and Beck-Nielsen, H. (2006). Impaired glycogen synthase activity and mitochondrial dysfunction in skeletal muscle; Markers or Mediators of insulin resistance in type 2 diabetes; Curr. Diabetes Rev 2; 375-395.

26. Hu, G., Barengo, N.C., Tuomilehto, J., Lakka, T.A., Nissinen A, Jousilahti P (2004). Relationship of physical activity and body mass index to the risk of hypertension: a prospective study in Finland; Hypertension; 43;25-30.

27. IDF Diabetes Atlas; International Diabetes Federation; Sixth edition; 2014 update.

28. Ivy, J.L. (1991). Muscle glycogen synthesis before and after exercise; Sports medicine 11; 6-19.

29. Jan Willem Van Dijk., Kyra Tummers., Coen D.A., Stehouwer., et al. (2012). Exercise therapy in type 2 diabetes- Is daily exercise required to optimize glycemic control?; Diabetes care; Vol 35.

30. Jan Willem Van Dijk., Maarten Venema., Willem Van Mechelen., et al. (2013). Effect of moderate intensity exercise versus activities of daily living on 24 hour blood glucose homeostasis in male patients with type 2 diabetes; Diabetes care; Vol 36.

31. Jergen Jensen., Per inge Rustad., Anders Jensen Kolnes., Yu- Chiang Lai. (2011). The role of skeletal muscle glycogen break down for regulation of insulin sensitivity by exercise; Frontiers in Physiology ; Volume 2; Article 112.

32. Jeukendrup, A.E., and Moseley, L. (2008). Multiple transportable carbohydrates enhance gastric emptying and fluid delivery; Scand J Med Sci Sports 2008; 20; 112-121.

33. Jeukendrup AE., and Gleeson, M. Sports nutrition; An introduction to energy production and performance; Human kinetics; Leeds UK 2010; PP 124-125.

34. Joseph T Dipiro., Robert L Talbert., Gary C Yee., Gary R Matzke., Barbara G Wells., Michael Posey, L. (1997). Pharmacotherapy- A Pathophysiologic approach; Seventh edition. 
35. Zierath, J.R., and Wallberg-Henriksson, H. (2002). From Receptor to Effector : Insulin signal transduction in skeletal muscle from type 2 diabetic patients; Annals of the New York Academy of Sciences; Vol 967; PP 120-134.

36. Karin M Nelson, Gayle Reiber, Edward J Boyko (2002). Diet and Exercise among Adults with Type 2 Diabetes; Diabetes care; Volume 25; Number 10.

37. Kevin E Yarasheski (2003). Exercise Aging and Muscle protein metabolism; Journal of Gerontology; Medical sciences 2003; Vol 58A; No:10, 918-922.

38. Kim, J.D., Yu, B.P., Mc Carter, R.J., Leesy, Herlihy, J.T. (1996). Exercise and Diet modulate cardiac lipid peroxidation and antioxidant defences; Free Radiac Biol Med 1996; 20 (1); 83-88.

39. Koopman, R., Beelen, M., Stellingwerff, T., Pennings, B., Saris, W.H., Kies, A.K., Kuipers, H., and Van loon, L.J.C. (2007). Coingestion of carbohydrate with protein does not further augment post exercise muscle protein synthesis, American Journal of Physiology Endocrinology and Metabolism 293; E833-842.

40. Krentz, A.J. (2003). Lipo protein abnormalities and their consequences for patients with type 2 diabetes; Diabetes Obes Metab 2003; 5: S19-S27.

41. Lorenzo A Gordon., Errol Y Morrison., Donovan, A., Mc Growder, et al. (2008). Effect of exercise therapy on lipid profile and oxidative stress indicators in patients with type 2 diabetes; BMC complementary and alternative medicine 2008; 8:21.

42. Louis Bherer., Kirk I Erickson., Teresa Liu-Ambrose. (2013). A Review of the effects of physical activity and exercise on cognitive and brain functions in older adults; Journal of aging Reasearch; Volume 2013; Article ID 657508 .

43. Maehlum, S., Hostmark, A.T., and Hermansen, L. (1977). Synthesis of muscle glycogen during recovery after prolonged severe exercise in diabetic and non diabetic subjects; Scandinavian Journal of clinical and laboratory investigation 37; 309-316.

44. Manisha chandalia., Abhimanyu Garg., Dieter Lut Johann. (2000). Beneficial effects of high dietary fiber intake in patients with type 2 diabetes mellitus; The new England Journal of medicine; 1392.

45. Bajaj, M., and Defronzo, R.A. (2003). Metabolic and molecular basis of insulin resistance; Journal of nuclear cardiology; Vol 10; No:3, PP 311-323.

46. Michael J Joyner, and Daniel J Green. (2009). Exercise protects the cardio vascular system; Effects beyond traditional risk factors; J Physiol 587; 23; PP 5551-5558.

47. Michael J Ormsbee, Christopher W Bach and Daniel A Baur; Pre-exercise nutrition: The role of macro nutrients, modified starches and supplements on metabolism and endurance performance; Nutrients 2014; 6; 1782-1808.

48. Muhammed, A., Abdul Ghani, and Ralph A Defronzo. (2010). Pathogenesis of insulin resistance in skeletal muscle; Journal of Biomedicine and Bio technology; Vol 2010; Article ID 476279.

49. Nadia Lascar., Amy Kennedy., Nikki Jackson, et al. (2013) Exercise to preserve beta cell function in recent onset type 1 diabetes mellitus (EXTOD)-a study protocol for a pilot randomized controlled trial; Trials 2013; $14: 180$.

50. Nicolucci, A., Balducci, S., Cardelli, P. et al. (2012). Italian Diabetes Exercise Study Investigators; Relationship of exercise volume to improvements of quality of life with supervised exercise training in patients with type 2 diabetes in a randomised controlled trial: the Italian Diabetes Exercise Study (IDES); Diabetologia 2012; 55; 579-588.

51. Niels Ortenblad., Hakan Westerblad., and Joachim Nielsen. (2013). Muscle glycogen stores and fatigue; The Journal of Physiology; 251629.

52. Thomas, N., Alder, E., Leese, G.P. (2015). Barriers to physical activity in patients with diabetes.

53. Patel, A, Mac mohan, S, Chalmers, .J. et al. (2007). Effects of a fixed combination of perindropil and indapamide on macro vascular and micro vascular outcomes in patients with type 2 diabetes mellitus ( the advance trial): a randomised controlled trial; Lancet 370; 829-840.

54. Pate, R.R., Pratt, M., Blair, S.N., Haskell, W.L., Macera, C.A., Bouchard, C. et al. (1995). Physical activity and Public Health; a recommendation from the centers for disease control and prevention and the American college of sports medicine; J Am Med Assoc 1995; 273; 402-7.

55. Paul T Williams., and Paul D Thompson. (2013). Walking Vs Running for hypertension, cholesterol and Diabetes risk reduction; Arterioscler Thromb Vasc Biol; 33 (5); 1085-1091.

56. Pedersen BK, Saltin B; Evidence for prescribing exercise as therapy in chronic disease, Scand J Med Sci Sports 2006; 16 ( Suppl 1): 3-63.

57. Pier Paolo De Feo, and Peter Schwarz. (2013). Is physical exercise a core therapeutical element for most patients with type 2 diabetes?; Diabetes care; Volume 36; Supplement 2.

58. Poehlman, E.T., Dvorak, P.V., Denino, W.F., Brochu, M., Ades, P.A. (2000). Effects of resistance training and endurance training on insulin sensitivity in non obese young women; a controlled randomized trial; J Clin Endocrinol Metab 85; 2463-2468.

59. Ross, R, Dagnone, D., Jones, P.J., Smith, H., Paddags, A. et al. (2000); Reduction in obesity and related co morbid conditions after Diet induced weight loss or exercise induced weight loss in men: a randomized controlled trial Ann Intern Med 133; 92-103. 
60. Sacks, F.M., Svetkey, L.P., Vollmer, W.M., et al. (2001). Effects of blood pressure of reduced dietary sodium and the dietary approaches to stop hypertension (DASH) Diet; N Engl J Med 2001; 344:3-10.

61. Saeid Golbidi and Ismail Laher, (2012). Exercise and cardio vascular system; Cardiology research and practice; Volume 2012; Article ID 210852.

62. Schellenburg, E.S., Dryden, D.M., Vander meer, B., Ha, C., Korowynk, G. (2013). lifestyle interventions for patients with and at risk for type 2 diabetes; a systematic review and meta analysis; Ann Intern Med 159; 543-551.

63. Praet S.F.E., Van Rooij, E.S.J., Wijtvliet, A. (2008). Brisk walking compared with an individualised medical fitness programme for patients with type 2 diabetes; a randomised controlled trial; Diabetologia 51; 736-746.

64. Shanhu Qiu., Xue Cai., Uwe Schumann., Martina velders., Zillin sun., and Jurgen Michael Steinacker. (2014). Impact of walking on glycemic control and other cardio vascular risk factors in type 2 diabetes; A Meta analysis; Plos One; Volume 9; Issue 10; e109767.

65. Shasi K Agarwal. (2012). Cardio vascular benefits of exercise; International Journal of General Medicine;5; $541-545$.

66. Shi, X., Summers, R.W., Schedl, H.P., Flanangan, S.W., Chang, R., and Gisolfi, C.V. (1995). Effects of carbohydrate type and concentration and solution osmolality on water absorption; Med Sci Sports Exerc, 27, 1607-1615.

67. Sheri R Colberg., Ronald J Sigal., Bo Fern hall., et al. (2010). Exercise and type 2 diabetes; Diabetes care; Vol 33; No 12.

68. Shulman, G.I., Rothman, D.L., Jue, T., Stein, P., Defronzo, R.A., and Shulman, R.G. (1990). Quantification of muscle glycogen synthesis in normal subjects and subjects with non insulin dependent diabetes by $13 \mathrm{C}$ nuclear magnetic resonance spectroscopy; N Engl J Med 322; 223-228.

69. Sigal, R.J., Kenny, G.P., Boul, C.N.G., Wells, G.A., Prud home, D. et al. (2007). Effects of aerobic training, resistance training or both on glycemic control in type 2 diabetes; Ann Intern Med 117; 357-369.

70. Stewart, K.J. (2002). Exercise training and the cardio vascular consequences of type 2 diabetes and hypertension; Plausible mechanisms for improving cardio vascular health; JAMA 288; 1622-1631.

71. Stephan, F.E Praet, Luc JC, Van loon; Exercise therapy in type 2 diabetes; Acta Diabetol (2009) 46: $263-278$.

72. Taylor, R., Magnusson, I., Rothman,, D.L., Cline, G.W., Caumo, A., Cobelli, C., and Shulman, G.I. (1996). Direct assessment of liver glycogen storage by $13 \mathrm{C}$ nuclear magnetic resonance spectroscopy and regulation of glucose homeostasis after a mixed meal in normal subjects; J Clin Invest 1996-97; 126-132.

73. Thomas, D.E., Elliot, E.J., Naughton, G.A. (2006). Exercise for type 2 diabetes mellitus; Cochrane Database Syst Rev 3; CD 002968.

74. Tuomilehto, J., Lindstom, J., Erikkson, J.G., et al. (2001). Prevention of type 2 diabetes mellitus by changes in lifestyle among subjects with impaired glucose tolerance; N Engl J Med 2001; 344; 1343-1350.

75. Tyler, C., Smith, M.S., Deborah, L., et al. (2007). Walking provides strong protection from cardio vascular disease mortality in older adults with diabetes; J Clin Epidemiol; 60(3): 309-317.

76. Wanson, J.C., and Drochman, P. (1968). Rabbit skeletal muscle glycogen- a morphological and biochemical study of glycogen beta particles isolated by precipitation- centrifugation method ; J Cell Biol 38; 130-150.

77. Zar chi thent., Srijit Das., and Leonard Joseph henry. (2013). Role of exercise in the management of diabetes mellitus;: the global scenario; Plos One; Volume 8; Issue 11; e80436.

78. Zhuo Fu., Elizabeth R Gilbert., and Dongmin Liu. (2013). Regulation of insulin synthesis and secretion and pancreatic beta cell dysfunction in Diabetes; Curr Diabetes Rev 2013; Jan 1; 9(1); 25-53. 